\title{
Immersion and Invariance Control for an Antagonistic Joint with Nonlinear Mechanical Stiffness
}

\author{
Thomas Wimböck, Christian Ott and Gerd Hirzinger \\ *Institute of Robotics and Mechatronics, German Aerospace Center (DLR), Germany, Email: thomas.wimboeck@dlr.de
}

\begin{abstract}
Tendon controlled robots with nonlinear mechanical tendon stiffness are becoming more and more popular. With the appropriate actuation, the joint position and the stiffness between motor and link side can be prescribed at the same time. In this paper, the modeling of tendon-driven elastic systems with nonlinear couplings is recapitulated. Based on the Immersion and Invariance (I\&I) framework a control law is developed that takes trajectories of the desired joint position and the pretension as input. The contribution of this paper are the application of the I\&I framework to tendon-controlled systems with variable stiffness which requires to consider the internal degrees of freedom to realize the pretension, respectively the mechanical joint stiffness. But also, and even more importantly, the handling of nonlinear transmission elements between the motor and the joint link is included. The performance and sensitivity is analyzed by simulating an antagonistically driven robot joint.
\end{abstract}

\section{INTRODUCTION}

The antagonistic tendon actuation principle from biology can be applied to robots by using tendons that connect the joints with the motors allowing a relocation of the drives to the base of the robot system. This design furthermore reduces the inertia of the robot system leading to faster motion while preserving safety for the human and the robot. A limitation in such systems is that excessively large tendon forces might occur in case of stiff tendons, which in the worst case leads to their breaking. The introduction of elastic elements into the tendon path relaxes this problem. This was done in some developments using linear tendon stiffness and by the introduction of variable stiffness (VS) elements [1][6]. Another example is the DLR hand arm system depicted in Figure 1 [7]. One of the first commercially available light-weight arms, namely the WAM [8], [9], is also based on tendon actuation. Controllers were presented that are capable of keeping the tendon tension while moving the robot joints with linear tendon stiffness [10]-[13]. A serial stiffness acts furthermore as mechanical low-pass filter to external forces, e.g. in case of a collision. In contrast to a constant elastic element, a variable stiffness allows to adapt the joint compliance to a given manipulation task, like for catching a heavy object in order to prevent damage of the robot or for fast assembly of parts. Another feature is that the serially connected springs act as energy storage that can be applied to e.g. a throwing task through transferring the stored spring energy to kinetic energy and vice versa [6], [14]. Furthermore, in [15] an impedance control law for a VS joint was developed. A tracking controller has been proposed and has been validated using a linearly coupled mechanism through experiments [16]. In [5] Palli et al. studied the

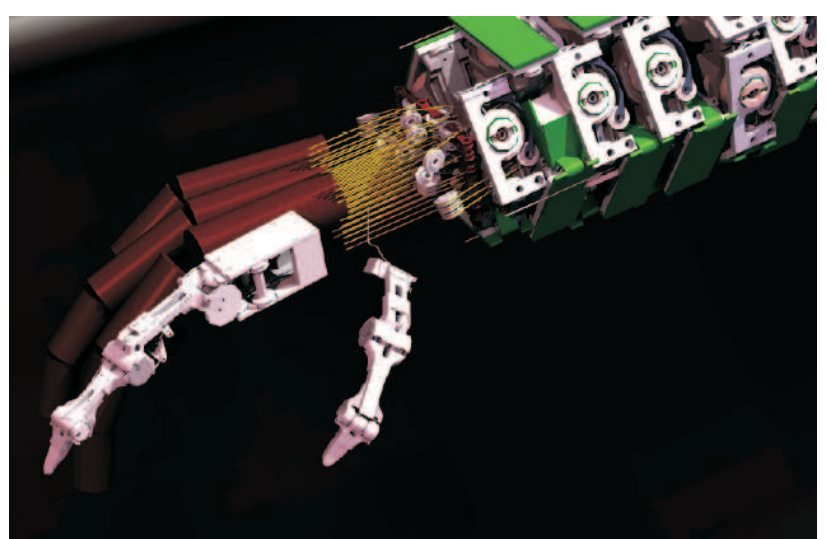

Fig. 1. CAD drawing of the DLR hand arm system [7].

feedback linearization of uncoupled joints that are each driven antagonistically. Furthermore, an adaptive motor level PD controller was presented in [17] for a single antagonistic joint that was verified by means of experiments. Passivitybased impedance controllers for flexible joint robots were presented by Ott and Albu-Schäffer [18], [19]. In this framework, the emphasis was to derive controllers from potential functions and to develop control laws that realize a predefined stiffness characteristics in the joints by means of control. In [20] we presented a particular PD controller that can set joint positions and joint stiffness independently in a robust fashion. However, since the controller was only based on the motor positions, the transient behavior could not fully be specified. The contribution of this paper are the application of the I\&I framework [21] to tendon-controlled systems with variable stiffness which requires the handling of nonlinear transmission elements between the motor and the joint link, and to consider the internal degrees of freedom that are related to pretension, respectively effective mechanical joint stiffness.

In the second section we will first review the I\&I stabilization theorem as proposed by Astolfi et al. in [21]. The modeling of an antagonistic joint with nonlinear tendon stiffness is described in section 3, while the I\&I control is applied in section 4 . The developed controller is compared in simulation with a motor PD controller in section 5.

\section{I\&I STABILIZATION}

This section introduces the I\&I framework with the main theorem proposed by Astolfi et al. in [21]. 
Theorem 1: Consider the system

$$
\dot{\boldsymbol{x}}=\boldsymbol{f}(\boldsymbol{x})+\boldsymbol{g}(\boldsymbol{x}) \boldsymbol{u},
$$

with $\boldsymbol{x} \in \mathbb{R}^{n}, \boldsymbol{u} \in \mathbb{R}^{m}$, and an equilibrium point $\boldsymbol{x}^{*} \in \mathbb{R}^{n}$ to be stabilised. Assume that there exist smooth mappings $\boldsymbol{\alpha}: \mathbb{R}^{p} \rightarrow \mathbb{R}^{p}, \boldsymbol{\pi}: \mathbb{R}^{p} \rightarrow \mathbb{R}^{n}, \boldsymbol{\phi}: \mathbb{R}^{n} \rightarrow \mathbb{R}^{n-p}, \boldsymbol{c}: \mathbb{R}^{p} \rightarrow$ $\mathbb{R}^{m}$ and $\boldsymbol{v}: \mathbb{R}^{n \times(n-p)} \rightarrow \mathbb{R}^{m}$, with $p<n$, such that the following hold.

1) The target system

$$
\dot{\xi}=\alpha(\xi),
$$

with $\boldsymbol{\xi} \in \mathbb{R}^{p}$ has a globally asymptotically stable equilibrium $\boldsymbol{\xi}^{*} \in \mathbb{R}^{p}$ and

$$
\boldsymbol{x}^{*}=\boldsymbol{\pi}\left(\boldsymbol{\xi}^{*}\right) .
$$

2) For all $\boldsymbol{\xi} \in \mathbb{R}^{p}$,

$$
\boldsymbol{f}(\boldsymbol{\pi}(\boldsymbol{\xi}))+\boldsymbol{g}(\boldsymbol{\pi}(\boldsymbol{\xi})) \boldsymbol{c}(\boldsymbol{\pi}(\boldsymbol{\xi}))=\frac{\partial \boldsymbol{\pi}}{\partial \boldsymbol{\xi}} \boldsymbol{\alpha}(\boldsymbol{\xi}) .
$$

3) The set identity

$$
\left\{\boldsymbol{x} \in \mathbb{R}^{n} \mid \boldsymbol{\phi}(\boldsymbol{x})=\mathbf{0}\right\}=\left\{\boldsymbol{x} \in \mathbb{R}^{n} \mid \boldsymbol{x}=\boldsymbol{\pi}(\boldsymbol{\xi}), \boldsymbol{\xi} \in \mathbb{R}^{p}\right\}
$$

holds.

$$
\begin{aligned}
\dot{z} & =\frac{\partial \phi}{\partial \boldsymbol{x}}(\boldsymbol{f}(\boldsymbol{x})+\boldsymbol{g}(\boldsymbol{x}) \boldsymbol{v}(\boldsymbol{x}, \boldsymbol{z})) \\
\dot{\boldsymbol{x}} & =\boldsymbol{f}(\boldsymbol{x})+\boldsymbol{g}(\boldsymbol{x}) \boldsymbol{v}(\boldsymbol{x}, \boldsymbol{z}),
\end{aligned}
$$

are bounded and (5) has a uniformly globally asymptotically stable equilibrium at $\boldsymbol{z}=\mathbf{0}$.

Then $\boldsymbol{x}^{*}$ is a globally asymptotically stable equilibrium of the closed-loop system

$$
\dot{\boldsymbol{x}}=\boldsymbol{f}(\boldsymbol{x})+\boldsymbol{g}(\boldsymbol{x}) \boldsymbol{v}(\boldsymbol{x}, \phi(\boldsymbol{x})) .
$$

Taken from [21], this theorem can be interpreted in the following way. The objective is to find a manifold $\mathcal{M}=$ $\left\{\boldsymbol{x} \in \mathbb{R}^{n} \mid \boldsymbol{x}=\boldsymbol{\pi}(\boldsymbol{\xi}), \boldsymbol{\xi} \in \mathbb{R}^{p}\right\}$ based on the system (1) and the target dynamics (2). This manifold can be rendered invariant and asymptotically stable, and such that the well-defined restriction of the closed-loop system to $\mathcal{M}$ is described by the target system (condition 3). Note that the control input $\boldsymbol{u}$ that makes the manifold invariant is not unique, since it is uniquely defined only on $\mathcal{M}$. One possible control, that drives the off-the-manifold coordinates $z$ to zero and keeps the system bounded, is selected. The I\&I concept is illustrated for $p=2$ and $n=3$ in Fig. 2.

\section{Modeling OF AN ANTAGONISTIC JOINT WITH NONLINEAR TENDON STIFFNESS}

In Fig. 3, a simple tendon-network consisting of one joint and two tendons connected by nonlinear springs is shown. In Table I, the variables to describe the equations of motion of an antagonistic robot joint are given. The tendon inverse

\begin{tabular}{|c|c|c|c|}
\hline$q$ & $\epsilon$ & $\mathbb{R}$ & Joint position \\
\hline$\theta$ & $\epsilon$ & $\mathbb{R}^{2}$ & 2 Motor positions \\
\hline $\boldsymbol{h}_{q}(\boldsymbol{q})$ & $\epsilon$ & $\mathbb{R}^{2}$ & 2 Tendon length changes w. r. t. joints \\
\hline$M$ & $\epsilon$ & $\mathbb{R}$ & Joint inertia \\
\hline$M_{\theta}$ & $\epsilon$ & $\mathbb{R}^{2 \times 2}$ & $\begin{array}{l}\text { P. d. effective tendon inertia matrix } \\
\text { including motor inertia }\end{array}$ \\
\hline$\tau_{\text {ext }}$ & $\epsilon$ & $\mathbb{R}$ & External torque \\
\hline $\boldsymbol{f}_{m}$ & $\in$ & $\mathbb{R}^{2}$ & Tendon motor forces (control input) \\
\hline $\boldsymbol{f}_{t}$ & $\epsilon$ & $\mathbb{R}^{2}$ & Tendon forces \\
\hline $\boldsymbol{f}_{f, \theta}$ & $\epsilon$ & $\mathbb{R}^{2}$ & Motor friction forces \\
\hline
\end{tabular}
kinematics $\boldsymbol{h}_{q}(\boldsymbol{q})$ gives the motor positions as a function of

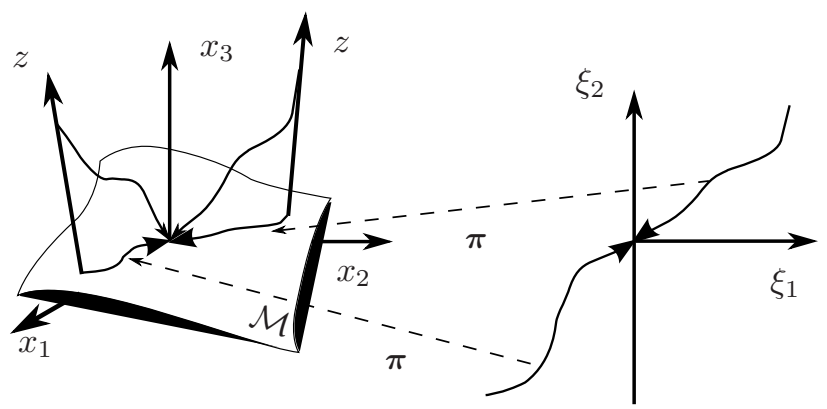

Fig. 2. Illustration of the immersion and invariance approach according to [21].

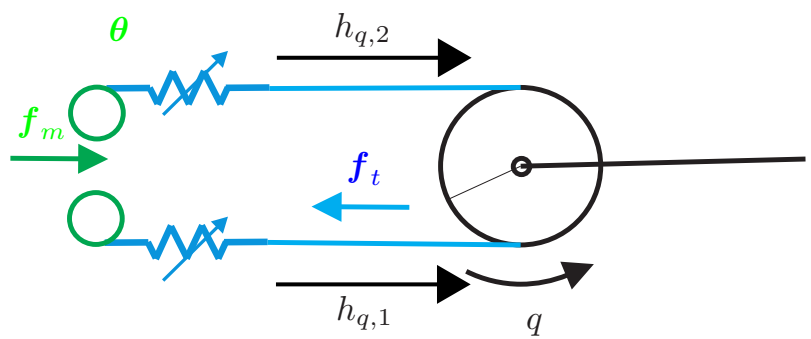

Fig. 3. Simple example of an antagonistic joint with two tendons connected via nonlinear springs to the joint.

TABLE I

DEFINITION OF VARIABLES.

the joint angles $\boldsymbol{q}$. The function $\boldsymbol{h}_{q}(\boldsymbol{q})$ can be used to derive a differential map $\boldsymbol{P}(\boldsymbol{q})$ :

$$
\boldsymbol{P}(\boldsymbol{q})=\left(\frac{\partial \boldsymbol{h}_{q}(\boldsymbol{q})}{\partial \boldsymbol{q}}\right)^{T} .
$$

In the robotics literature, this map is also known as coupling matrix [22]. Note that in contrast to the Jacobian matrix of a serial kinematic chain, the transposed coupling matrix maps from joint to tendon velocities

$$
\dot{\boldsymbol{h}}_{q}=\boldsymbol{P}^{T}(\boldsymbol{q}) \dot{\boldsymbol{q}}
$$

and the tendon forces are related to the joint torques by

$$
\boldsymbol{\tau}_{q}=\boldsymbol{P}(\boldsymbol{q}) \boldsymbol{f}_{t} .
$$

For a well-designed mechanism, the coupling matrix $\boldsymbol{P}(\boldsymbol{q})$ has full row rank over the whole configuration space [23].

Using the coupling matrix $\boldsymbol{P}(\boldsymbol{q})$, it is straightforward to formulate the equations of motion for an antagonistic joint 
[22]:

$$
\begin{aligned}
M \ddot{q} & =\boldsymbol{P}(q) \boldsymbol{f}_{t}(\boldsymbol{q}, \boldsymbol{\theta})+\tau_{e x t} \\
\boldsymbol{M}_{\theta} \ddot{\boldsymbol{\theta}}+\boldsymbol{f}_{f, \theta} & =\boldsymbol{f}_{m}-\boldsymbol{f}_{t}(\boldsymbol{q}, \boldsymbol{\theta}),
\end{aligned}
$$

with the inertia matrix of the motor side $\boldsymbol{M}_{\theta}$ being a diagonal matrix. A joint with linear coupling is considered, i.e.

$$
\begin{aligned}
\boldsymbol{h}_{q}(q) & =\boldsymbol{P}^{T} q, \\
\boldsymbol{P} & =[1,-1],
\end{aligned}
$$

This represents the most simple antagonistic system and as nonlinear transmission element the exponential tendon force characteristics

$$
f_{t, i}\left(q, \theta_{i}\right)=k_{t, i}\left(e^{\gamma_{i}\left(-\left(\boldsymbol{P}^{T} q\right)_{i}+\theta_{i}\right)}-1\right), \quad \forall i=1,2,
$$

is used [17]. This characteristics is parameterized by $\gamma=$ $\left(\gamma_{1}, \gamma_{2}\right)^{T}$ and $\boldsymbol{k}_{t}=\left(k_{t, 1}, k_{t, 2}\right)^{T}$. The vector $\boldsymbol{f}_{t}(q, \boldsymbol{\theta})=$ $\left(f_{t, 1}\left(q, \theta_{1}\right), f_{t, 2}\left(q, \theta_{2}\right)\right)^{T}$ contains the forces in stacked notation. Note that this choice of force characteristics does not limit the applicability of the I\&I control to other functions, as long they are strictly increasing ensuring a unique inverse solution. With the state $\boldsymbol{x}^{T}=(q, \dot{q}, \boldsymbol{\theta}, \dot{\boldsymbol{\theta}})$, assigning $x_{1}=q$, $\boldsymbol{x}_{3}=\boldsymbol{\theta}$, and $\boldsymbol{u}=\boldsymbol{f}_{m}$ the following state space model is obtained

$$
\begin{aligned}
& \dot{x}_{1}=x_{2} \\
& \dot{x}_{2}=M^{-1}\left(\boldsymbol{P} \boldsymbol{f}_{t}\left(x_{1}, \boldsymbol{x}_{3}\right)+\tau_{\text {ext }}\right) \\
& \dot{\boldsymbol{x}}_{3}=\boldsymbol{x}_{4} \\
& \dot{\boldsymbol{x}}_{4}=-\boldsymbol{M}_{\theta}^{-1}\left(\boldsymbol{f}_{f, \theta}+\boldsymbol{f}_{t}\left(x_{1}, \boldsymbol{x}_{3}\right)-\boldsymbol{u}\right) .
\end{aligned}
$$

Rewriting this system as $\dot{\boldsymbol{x}}=\boldsymbol{l}(\boldsymbol{x})$ and setting the inputs $\tau_{\text {ext }}=0, \boldsymbol{u}=\mathbf{0}$ it can be computed easily that $\boldsymbol{l}(\boldsymbol{x})$ is locally Lipschitz on $\mathbb{R}^{6}$. With the physical interpretation that the system equation models two bodies that are connected by springs and one body dissipates energy by a friction term, it is clear that it is possible to find a compact subset for any initial condition, which contains the solution. Therefore, it is concluded that the equation has a unique solution for $t>0$.

\section{I\&I CONTROL OF AN ANTAGONISTIC JOINT}

\section{A. Specification of the target dynamics}

The target system $\boldsymbol{\alpha}(\boldsymbol{\xi}, t)$ is chosen to prescribe a desired impedance behavior to the link side dynamics of the robot. In this way, controllers that exist in various forms can be used for this reduced order system. The I\&I concept [21] is used to robustify against the higher-order dynamics of the motors side. This means that the motor dynamics that are faster than the link dynamics are not considered in the target system. The structure of the target system $\boldsymbol{\alpha}(\boldsymbol{\xi}, t)$ hence resembles the link dynamics in which a controller for rigid robots $\bar{w}(\boldsymbol{\xi}, t)$ can be integrated, i.e.

$$
\begin{aligned}
& \dot{\xi}_{1}=\xi_{2} \\
& \dot{\xi}_{2}=-M^{-1}\left(\bar{w}(\boldsymbol{\xi}, t)-\tau_{e x t}\right),
\end{aligned}
$$

with $\xi \in \mathbb{R}^{2}$. A PD controller plus feedforward terms is considered $^{1}$ that is given as

$$
\bar{w}(\boldsymbol{\xi}, t)=K_{p}\left(\xi_{1}-\xi_{1}^{*}(t)\right)+K_{d}\left(\xi_{2}-\dot{\xi}_{1}^{*}(t)\right)+M \ddot{\xi}_{1}^{*}(t),
$$

\footnotetext{
${ }^{1}$ Certainly, the tracking controller proposed by Slotine and Li [24] could be applied as well.
}

with $K_{p}, K_{d}>0, \xi_{1}^{*}(t) \in \mathcal{C}^{4}$.

\section{B. Computation of the map $\boldsymbol{\pi}(\boldsymbol{\xi}, t)$}

A major difficulty using the I\&I framework is to solve the partial differential equation (3). Since $\bar{w}(\boldsymbol{\xi}, t)$ is an explicit function of time, additionally the term $\frac{\partial \pi}{\partial t}$ has to be included

$$
\boldsymbol{f}(\boldsymbol{\pi}(\boldsymbol{\xi}, t))+\boldsymbol{g}(\boldsymbol{\pi}(\boldsymbol{\xi}, t)) \boldsymbol{c}(\boldsymbol{\pi}(\boldsymbol{\xi}, t))=\frac{\partial \boldsymbol{\pi}}{\partial \boldsymbol{\xi}} \boldsymbol{\alpha}(\boldsymbol{\xi}, t)+\frac{\partial \boldsymbol{\pi}}{\partial t} .
$$

Since the target system (16) resembles the link side dynamics of the original system (15), the solution to this equation can be obtained by starting to set $\pi_{1}(\boldsymbol{\xi})=\xi_{1}$. Then, looking at $\dot{x}_{1}=x_{2}$ it follows directly that $\pi_{2}(\boldsymbol{\xi})=\xi_{2}$. Based on the term $\dot{x}_{2}$, the mapping $\boldsymbol{\pi}_{3}(\boldsymbol{\xi}, t)=\left(\pi_{3,1}(\boldsymbol{\xi}, t), \pi_{3,2}(\boldsymbol{\xi}, t)\right)^{T}$ is derived as

$$
\dot{x}_{2}=\frac{\partial \pi_{2}(\boldsymbol{\xi})}{\partial \boldsymbol{\xi}} \dot{\boldsymbol{\xi}}
$$

using the systems (15) and (16)

$$
M^{-1}\left(\boldsymbol{P} \boldsymbol{f}_{t}\left(\xi_{1}, \boldsymbol{\pi}_{3}(\boldsymbol{\xi}, t)\right)+\tau_{\text {ext }}\right)=-M^{-1}\left(\bar{w}(\boldsymbol{\xi}, t)-\tau_{\text {ext }}\right),
$$

with $\boldsymbol{P}$ tendon-controllable [23] and therefore with full row rank. This equation is rewritten as

$$
\boldsymbol{f}_{t}\left(\xi_{1}, \boldsymbol{\pi}_{3}(\boldsymbol{\xi}, t)\right)=-\boldsymbol{P}^{+} \bar{w}(\boldsymbol{\xi}, t)-\mathcal{N}(\boldsymbol{P}) w_{n}(t)=-\boldsymbol{w}(\boldsymbol{\xi}, t) .
$$

with the nullspace basis of $\boldsymbol{P}, \mathcal{N}(\boldsymbol{P})=-\frac{\sqrt{2}}{2}[1,1]^{T}$, $w_{n}(t) \in \mathcal{C}^{2}$, and $\boldsymbol{w}(\boldsymbol{\xi}, t)$ the modified rigid body controller. This furthermore gives the possibility to use the nullspace of $\boldsymbol{P}$ to add feed-forward forces $w_{n}$ that shall realize pretension between the motors while at the same time not affecting the link motion. Note, that we assume in this paper that the pretension $w_{n}$ is set such that the pulling constraint for the tendons is fulfilled. In the case of nonlinear mechanical stiffness this will be used to specify a desired mechanical stiffness between the motors and the robot link [1], [20].

This set of nonlinear equations needs to be solved for $\boldsymbol{\pi}_{3}(\boldsymbol{\xi}, t)$. Since in each equation only the $i^{t h}$ component of $\pi_{3}(\boldsymbol{\xi}, t)$ is a function of the known terms, the solution will be derived componentwise. Using the force model (14), the $i^{t h}$ component of $\boldsymbol{\pi}_{3}(\boldsymbol{\xi}, t)$ can be computed to

$$
\boldsymbol{\pi}_{3, i}(\boldsymbol{\xi}, t)=\left(\boldsymbol{P}^{T} \xi_{1}\right)_{i}+\gamma_{i}^{-1} \ln \left(1-k_{t, i}^{-1} w_{i}(\boldsymbol{\xi}, t)\right) .
$$

The solution to $\pi_{3}(\boldsymbol{\xi})$ can then be given by stacking the components

$$
\boldsymbol{\pi}_{3}(\boldsymbol{\xi}, t)=\boldsymbol{P}^{T} \xi_{1}+\left(\begin{array}{c}
\gamma_{1}^{-1} \ln \left(1-k_{t, 1}^{-1} w_{1}(\boldsymbol{\xi}, t)\right) \\
\gamma_{2}^{-1} \ln \left(1-k_{t, 2}^{-1} w_{2}(\boldsymbol{\xi}, t)\right)
\end{array}\right) .
$$

The expression $\boldsymbol{\pi}_{3}(\boldsymbol{\xi})$ can be interpreted as virtual desired motor positions that are required to prescribe the target dynamics.

Using $\dot{\boldsymbol{x}}_{3}=\boldsymbol{x}_{4}$ appearing in the system equations (15) it is straightforward to derive the map $\boldsymbol{\pi}_{4}(\boldsymbol{\xi}, t)$, respectively its $i^{\text {th }}$ component as

$$
\boldsymbol{\pi}_{4, i}(\boldsymbol{\xi}, t)=\frac{\partial \boldsymbol{\pi}_{3, i}(\boldsymbol{\xi}, t)}{\partial \boldsymbol{\xi}} \dot{\boldsymbol{\xi}}+\frac{\partial \boldsymbol{\pi}_{3, i}(\boldsymbol{\xi}, t)}{\partial t},
$$


while keeping in mind that $\boldsymbol{w}(\boldsymbol{\xi}, t)$ is also a function of time, and with

$$
\frac{\partial \boldsymbol{\pi}_{3, i}(\boldsymbol{\xi}, t)}{\partial w_{i}}=\frac{-\gamma_{i}^{-1} k_{t, i}^{-1}}{1-k_{t, i}^{-1} w_{i}(\boldsymbol{\xi}, t)} .
$$

Note that the denominator becomes zero at $w_{i}(\boldsymbol{\xi}, t)=k_{t, i}$. This corresponds to the force $f_{t, i}\left(x_{1}, x_{3, i}\right)=-k_{t, i}$ which means that $x_{3, i}-\left(\boldsymbol{P} x_{1}\right)_{i} \rightarrow-\infty$, and is not relevant here since (positive) pulling forces are assumed. Together with the system equations (15) the solution can be computed as

$$
\begin{aligned}
\pi_{4, i}(\boldsymbol{\xi}, t)= & \left(\boldsymbol{P}^{T} \xi_{2}\right)_{i} \\
+ & \frac{\partial \boldsymbol{\pi}_{3, i}(\boldsymbol{\xi}, t)}{\partial w_{i}}\left(\frac{\partial w_{i}(\boldsymbol{\xi}, t)}{\partial \xi_{1}} \xi_{2}\right. \\
& -\frac{\partial w_{i}(\boldsymbol{\xi}, t)}{\partial \xi_{2}}\left(M^{-1}\left(\boldsymbol{P} \boldsymbol{w}(\boldsymbol{\xi}, t)-\tau_{\text {ext }}\right)\right) \\
& \left.+\frac{\partial w_{i}(\boldsymbol{\xi}, t)}{\partial t}\right) .
\end{aligned}
$$

\section{Derivation of the manifold $\phi(x, t)=\mathbf{0}$}

The next step is to find an implicit definition of the manifold $\phi(x, t) \in \mathbb{R}^{6-2}$, i.e.

$$
\phi(\boldsymbol{x}, t)=\left(\begin{array}{l}
\phi_{1}(\boldsymbol{x}, t) \\
\phi_{2}(\boldsymbol{x}, t)
\end{array}\right)=\left(\begin{array}{l}
\phi_{1,1}(\boldsymbol{x}, t) \\
\phi_{1,2}(\boldsymbol{x}, t) \\
\phi_{2,1}(\boldsymbol{x}, t) \\
\phi_{2,2}(\boldsymbol{x}, t)
\end{array}\right)
$$

With the direct correspondences of $\pi_{1}(\boldsymbol{\xi})=x_{1}, \pi_{2}(\boldsymbol{\xi})=x_{2}$, two equations of the manifold are constructed by

$$
\begin{aligned}
& \phi_{1}(\boldsymbol{x}, t)=\boldsymbol{x}_{3}-\left.\boldsymbol{\pi}_{3}(\boldsymbol{\xi}, t)\right|_{\xi_{1}=x_{1}, \xi_{2}=x_{2}} \\
& \phi_{1}(\boldsymbol{x}, t)=\boldsymbol{x}_{3}-\boldsymbol{P}^{T} x_{1}-\left(\begin{array}{c}
\gamma_{1}^{-1} \ln \left(1-k_{t, 1}^{-1} w_{1}(\boldsymbol{x}, t)\right) \\
\gamma_{2}^{-1} \ln \left(1-k_{t, 2}^{-1} w_{2}(\boldsymbol{x}, t)\right)
\end{array}\right) .
\end{aligned}
$$

In a similar fashion $\phi_{2}(\boldsymbol{x}, t)$ is constructed. First, $\overline{\boldsymbol{\phi}}_{2}(\boldsymbol{x}, t)$ is derived as

$$
\overline{\boldsymbol{\phi}}_{2}(\boldsymbol{x}, t)=\boldsymbol{x}_{4}-\left.\boldsymbol{\pi}_{4}(\boldsymbol{\xi}, t)\right|_{\xi_{1}=x_{1}, \xi_{2}=x_{2}} .
$$

Secondly, as pointed out in [21], the already obtained set equation $\phi_{1}(\boldsymbol{x}, t)=0$ is used. Instead of using this function directly we rewrite it as $\boldsymbol{w}(\boldsymbol{x}, t)=-\boldsymbol{f}_{t}\left(x_{1}, \boldsymbol{\pi}_{3}(\boldsymbol{x}, t)\right)$ that is inserted in $\overline{\boldsymbol{\phi}}_{2}(\boldsymbol{x}, t)$. In this way, we achieve that $\dot{\boldsymbol{\phi}}_{1}(\boldsymbol{x}, t)=$ $\phi_{2}(\boldsymbol{x}, t)$ which is helpful to simplify the controller design to render the manifold attractive. Note that even though the term $\boldsymbol{\pi}_{4}(\boldsymbol{\xi}, t)=\dot{\boldsymbol{\pi}}_{3}(\boldsymbol{\xi}, t)=\frac{\partial \boldsymbol{\pi}_{3}}{\partial \boldsymbol{\xi}} \dot{\boldsymbol{\xi}}$ appears in $\overline{\boldsymbol{\phi}}_{2}(\boldsymbol{x}, t)$ this not equal to $\phi_{2}(\boldsymbol{x}, t)=\dot{\boldsymbol{\phi}}_{1}(\boldsymbol{x}, t)=\frac{\partial \boldsymbol{\phi}_{1}}{\partial \boldsymbol{x}} \dot{\boldsymbol{x}}$, since $\boldsymbol{f}_{t}\left(x_{1}, \boldsymbol{\pi}_{3}(\boldsymbol{x}, t)\right)$ does not appear in the target dynamics. The term $\phi_{2}(\boldsymbol{x}, t)$ is obtained for index $i$ as

$$
\begin{aligned}
\phi_{2, i}(\boldsymbol{x}, t)= & \boldsymbol{x}_{4, i}-\tilde{\boldsymbol{\pi}}_{4, i}(\boldsymbol{x}, t), \\
\tilde{\boldsymbol{\pi}}_{4, i}(\boldsymbol{x}, t)= & \left(\boldsymbol{P}^{T} x_{2}\right)_{i}+\frac{\partial \boldsymbol{\pi}_{3, i}(\boldsymbol{x}, t)}{\partial w_{i}}\left(\frac{\partial w_{i}(\boldsymbol{x}, t)}{\partial x_{1}} x_{2}\right. \\
& +\frac{\partial w_{i}(\boldsymbol{x}, t)}{\partial x_{2}}\left(M^{-1}\left(\boldsymbol{P} \boldsymbol{f}_{t}\left(x_{1}, \boldsymbol{\pi}_{3}(\boldsymbol{x}, t)\right)+\tau_{e x t}\right)\right) \\
& \left.+\frac{\partial w_{i}(\boldsymbol{( x , t )}}{\partial t}\right) .
\end{aligned}
$$

Having defined the manifold $\phi(x, t)$, the equations of the off-the-manifold dynamics can be determined according to the modified equation (5)

$$
\dot{\boldsymbol{z}}=\frac{\partial \phi(\boldsymbol{x}, t)}{\partial \boldsymbol{x}}(\boldsymbol{f}(\boldsymbol{x})+\boldsymbol{g}(\boldsymbol{x}) \boldsymbol{v}(\boldsymbol{x}, \boldsymbol{z}, t))+\frac{\partial \phi(\boldsymbol{x}, t)}{\partial t} .
$$

The equation of $\dot{z}_{1}=z_{2}$ is obtained by the construction of $\phi(\boldsymbol{x}, t)$. With equation (29) the remaining system equations for $\dot{z}_{2}$ are determined for each index $i$ by differentiation as

$$
\begin{aligned}
\dot{\boldsymbol{z}}_{2, i}= & m_{i}(\boldsymbol{x}, t)+\left(\boldsymbol{M}_{\theta}^{-1}\right)_{i, i} \boldsymbol{u}_{i}, \\
m_{i}(\boldsymbol{x}, t)= & -\left(\boldsymbol{M}_{\theta}^{-1}\right)_{i, i}\left(f_{t, i}\left(x_{1}, \boldsymbol{\pi}_{3}(\boldsymbol{x}, t)\right)+f_{f, \theta, i}\right)-\left(\boldsymbol{P}^{T} \dot{x}_{2}\right)_{i}- \\
& \frac{d}{d t}\left\{\frac{\partial \boldsymbol{\pi}_{3, i}(\boldsymbol{x}, t)}{\partial w_{i}}\right\}\left(\frac{\partial w_{i}(\boldsymbol{x}, t)}{\partial x_{1}} x_{2}+\frac{\partial w_{i}(\boldsymbol{x}, t)}{\partial t}\right. \\
& \left.+\frac{\partial w_{i}(\boldsymbol{x}, t)}{\partial x_{2}}\left(M^{-1}\left(\boldsymbol{P} \boldsymbol{f}_{t}\left(x_{1}, \boldsymbol{\pi}_{3}(\boldsymbol{x}, t)\right)+\tau_{e x t}\right)\right)\right) \\
& -\frac{\partial \boldsymbol{\pi}_{3, i}(\boldsymbol{x}, t)}{\partial w_{i}}\left(\frac{\partial w_{i}(\boldsymbol{x}, t)}{\partial x_{1}} \dot{x}_{2}+\frac{\partial^{2} w_{i}(\boldsymbol{x}, t)}{\partial t^{2}}\right. \\
& \left.-\frac{\partial w_{i}(\boldsymbol{x}, t)}{\partial x_{2}}\left(M^{-1}\left(\boldsymbol{P} \dot{\boldsymbol{f}}\left(x_{1}, \boldsymbol{\pi}_{3}(\boldsymbol{x}, t)\right)+\dot{\tau}_{\text {ext }}\right)\right)\right) .
\end{aligned}
$$

The control law is then chosen as

$$
\boldsymbol{u}=\boldsymbol{v}(\boldsymbol{x}, \boldsymbol{z}, t)=-\boldsymbol{M}_{\theta}\left(\boldsymbol{m}(\boldsymbol{x}, t)+\boldsymbol{K}_{z, 1} \boldsymbol{z}_{1}+\boldsymbol{K}_{z, 2} \boldsymbol{z}_{2}\right),
$$

with $\boldsymbol{K}_{z, 1}, \boldsymbol{K}_{z, 2} \in \mathbb{R}^{2 \times 2}$ which are each positive-definite matrices such that the off-the-manifold dynamics are stabilized. Note that the $z_{1}$ term represents the error term between the position of the motors and the desired virtual motor positions of the target system. This means the stabilization of the off-the-manifold dynamics can be interpreted as an underlying motor position controller.

D. Discussion of the boundedness of the closed-loop system

In this paragraph the boundedness of the closed-loop equations with the states $(\boldsymbol{x}, \boldsymbol{z})$ is analyzed in order to show that condition 4 of the I\&I stabilization theorem holds. The closed loop equations are obtained by inserting the control law (31) in the system equation (15). Then, the (fast) coordinates $\boldsymbol{x}_{3}$ and $\boldsymbol{x}_{4}$ are replaced by the coordinate transformation $\phi_{1}=\boldsymbol{x}_{3}-\boldsymbol{\pi}_{3}(\boldsymbol{x}, t)$ and $\phi_{2}=\boldsymbol{x}_{4}-\tilde{\boldsymbol{\pi}}_{4}(\boldsymbol{x}, t)$. To complete the change of coordinates we need to solve for $\boldsymbol{x}_{3}=\phi_{1}+\boldsymbol{\pi}_{3}(\boldsymbol{x}, t)$ that is inserted into the remaining systems equations

$$
\begin{aligned}
\dot{\boldsymbol{z}}_{1} & =\boldsymbol{z}_{2} \\
\dot{\boldsymbol{z}}_{2} & =-\boldsymbol{K}_{z, 1} \boldsymbol{z}_{1}-\boldsymbol{K}_{z, 2} \boldsymbol{z}_{2} \\
\dot{x}_{1} & =x_{2} \\
\dot{x}_{2} & =M^{-1}\left(\boldsymbol{P} \boldsymbol{f}_{t}\left(x_{1}, \phi_{1}+\boldsymbol{\pi}_{3}(\boldsymbol{x}, t)\right)\right) \\
\dot{\phi}_{1} & =\boldsymbol{z}_{2} \\
\dot{\phi}_{2} & =-\boldsymbol{K}_{z, 1} \boldsymbol{z}_{1}-\boldsymbol{K}_{z, 2} \boldsymbol{z}_{2},
\end{aligned}
$$

while setting $\tau_{\text {ext }}=0$ to zero for this analysis. By construction, the off-the-manifold dynamics converge exponentially. Then, from equation (36) and (37) it can be seen that $\phi_{1}$ is bounded. Therefore, it remains to show that the set of equations (34) and (35) has a bounded solution. We therefore consider these equations as system with nonvanishing perturbation and follow Lemma 9.2 in [25]. The perturbation is generated by the term $\phi_{1}$. Setting $\phi_{1}=0$, the set of equations that represents the linear target dynamics is obtained. The nominal system is then given as

$$
\begin{aligned}
& \dot{x}_{1}=x_{2} \\
& \dot{x}_{2}=-M^{-1} \boldsymbol{P} \boldsymbol{w}(\boldsymbol{x}, t) .
\end{aligned}
$$


In order to bring the equations (34), (35) into a form to identify the total perturbation term $b_{2}\left(x_{1}, x_{2}, \phi_{1}, t\right)$ the term $M^{-1} \boldsymbol{P} \boldsymbol{w}(\boldsymbol{x}, t)$ from (39) is added and subtracted from equation (35) resulting in the perturbed system

$$
\begin{aligned}
\dot{x}_{1}= & x_{2} \\
\dot{x}_{2}= & -M^{-1} \boldsymbol{P} \boldsymbol{w}(\boldsymbol{x}, t)+b_{2}\left(x_{1}, x_{2}, \phi_{1}, t\right), \\
b_{2}\left(x_{1}, x_{2}, \phi_{1}, t\right)= & M^{-1} \boldsymbol{P} \boldsymbol{w}(\boldsymbol{x}, t) \\
& +M^{-1} \boldsymbol{P} \boldsymbol{f}_{t}\left(x_{1}, \phi_{1}+\boldsymbol{\pi}_{3}(\boldsymbol{x}, t)\right) .
\end{aligned}
$$

Together with the tendon force model (14), the definition of $\boldsymbol{\pi}_{3}(\boldsymbol{x}, t)$ in (22) (that contains the inverse operation on the tendon force model), and the identity $e^{a+b}=e^{a} e^{b}$, the perturbation term can be reformulated as

$b_{2}\left(x_{1}, x_{2}, \phi_{1}, t\right)=-M^{-1} \boldsymbol{P} \operatorname{diag}\{\boldsymbol{\delta}\} \boldsymbol{w}(\boldsymbol{x}, t)+M^{-1} \boldsymbol{P} \boldsymbol{K}_{t} \boldsymbol{\delta}$,

with $\delta_{i}=e^{\gamma_{i} \phi_{1, i}}-1$, for $i=1,2$, and the matrix $\boldsymbol{K}_{t}=\operatorname{diag}\left\{k_{t, 1}, k_{t, 2}\right\}$. Note, that the specific structure of the perturbation term is due to the particular choice of force characteristics. Note that as $\phi_{1} \rightarrow 0$ it follows that $b_{2} \rightarrow 0$.

The linear nominal system (38), (39) is transformed to the coordinates

$$
e_{1}=x_{1}-\xi_{1}^{*}(t), \quad e_{2}=x_{2}-\dot{\xi}_{1}^{*}(t) .
$$

With these coordinates and inserting (17) the nominal system becomes

$$
\begin{aligned}
& \dot{e}_{1}=e_{2} \\
& \dot{e}_{2}=-M^{-1}\left(K_{p} e_{1}+K_{d} e_{2}\right)
\end{aligned}
$$

For the nominal linear system there exists a Lyapunov function [25]

$$
\begin{aligned}
V(\boldsymbol{e})= & \boldsymbol{e}^{T} \boldsymbol{P}_{L} \boldsymbol{e}, \\
\text { with } & \boldsymbol{P}_{L} \boldsymbol{A}+\boldsymbol{A}^{T} \boldsymbol{P}_{L}=-\boldsymbol{Q}=-\mathbf{I} \\
\text { and } & \boldsymbol{A}=\left[\begin{array}{cc}
0 & 1 \\
-M^{-1} K_{p} & -M^{-1} K_{d}
\end{array}\right],
\end{aligned}
$$

with $e=\left(e_{1}, e_{2}\right)^{T}$. This Lyapunov function will be used as a candidate potential function for the perturbed system (34), (35) for which we want to show boundedness. As intermediate step, the perturbation term $b_{2}(41)$ is rewritten in the coordinates $\boldsymbol{e}$, i.e.

$$
\begin{aligned}
b_{2}\left(\boldsymbol{e}, \phi_{1}, t\right)= & -M^{-1} \boldsymbol{P} \operatorname{diag}\{\boldsymbol{\delta}\} \boldsymbol{P}^{+}\left(K_{p} e_{1}+K_{d} e_{2}\right) \\
& +M^{-1} \boldsymbol{P}\left(\boldsymbol{K}_{t} \boldsymbol{\delta}-\operatorname{diag}\{\boldsymbol{\delta}\} \boldsymbol{P}^{+} M \ddot{\xi}_{1}^{*}\right)
\end{aligned}
$$

The derivative of $V(e)$ along the trajectories of the perturbed system satisfies

$$
\dot{V}(\boldsymbol{e}, t)=-\|\boldsymbol{e}\|^{2}+\boldsymbol{e}^{T} \boldsymbol{K}_{\delta} \boldsymbol{e}+2 \boldsymbol{e}^{T} \boldsymbol{P}_{L} \overline{\boldsymbol{\delta}},
$$

with $\overline{\boldsymbol{\delta}}=\left(0, M^{-1} \boldsymbol{P}\left(\boldsymbol{K}_{t} \boldsymbol{\delta}-\operatorname{diag}\{\boldsymbol{\delta}\} \boldsymbol{P}^{+} M \ddot{\xi}_{1}^{*}\right)\right), \boldsymbol{K}_{\delta}=$ $\boldsymbol{P}_{L} \boldsymbol{A}_{\delta}+\boldsymbol{A}_{\delta}^{T} \boldsymbol{P}_{L}$, and

$\boldsymbol{A}_{\delta}=\left[\begin{array}{cc}0 & 0 \\ -M^{-1} \boldsymbol{P} \operatorname{diag}\{\boldsymbol{\delta}\} \boldsymbol{P}^{+} K_{p} & -M^{-1} \boldsymbol{P} \operatorname{diag}\{\boldsymbol{\delta}\} \boldsymbol{P}^{+} K_{d}\end{array}\right]$.

For the quadratic perturbation term the inequality

$$
\boldsymbol{e}^{T} \boldsymbol{K}_{\delta} \boldsymbol{e} \leq \lambda_{\max }\left(\boldsymbol{K}_{\delta}\right)\|\boldsymbol{e}\|^{2}
$$

can be used. For the second (indefinite) term the CauchySchwarz inequality $a^{T} b \leq\|a\|\|b\|$ is applied that gives

$$
\begin{aligned}
2 \boldsymbol{e}^{T} \boldsymbol{P}_{L} \overline{\boldsymbol{\delta}} & \leq 2\left\|\boldsymbol{e}^{T} \boldsymbol{P}_{L}\right\|\|\overline{\boldsymbol{\delta}}\| \\
& \leq 2 \lambda_{\max }\left(\boldsymbol{P}_{L}\right)\|\boldsymbol{e}\|\|\overline{\boldsymbol{\delta}}\|
\end{aligned}
$$

Using these two inequalities we obtain

$$
\dot{V}(\boldsymbol{e}, t) \leq-\|\boldsymbol{e}\|^{2}+\lambda_{\max }\left(\boldsymbol{K}_{\delta}\right)\|\boldsymbol{e}\|^{2}+2 \lambda_{\max }\left(\boldsymbol{P}_{L}\right)\|\boldsymbol{e}\|\|\overline{\boldsymbol{\delta}}\|,
$$

Suppose $\lambda_{\max }\left(\boldsymbol{K}_{\delta}\right) \leq(1-\zeta), 0<\zeta<1^{2}$, we can write

$$
\begin{aligned}
\dot{V}(\boldsymbol{e}, t) & \leq-\|\boldsymbol{e}\|^{2}+(1-\zeta)\|\boldsymbol{e}\|^{2}+2 \lambda_{\max }\left(\boldsymbol{P}_{L}\right)\|\boldsymbol{e}\|\|\overline{\boldsymbol{\delta}}\|(51) \\
& =-\zeta\|\boldsymbol{e}\|^{2}+2 \lambda_{\max }\left(\boldsymbol{P}_{L}\right)\|\boldsymbol{e}\|\|\overline{\boldsymbol{\delta}}\| \\
& =-(1-\nu) \zeta\|\boldsymbol{e}\|^{2}-\nu \zeta\|\boldsymbol{e}\|^{2}+2 \lambda_{\max }\left(\boldsymbol{P}_{L}\right)\|\boldsymbol{e}\|\|\overline{\boldsymbol{\delta}}\| \\
& =-(1-\nu) \zeta\|\boldsymbol{e}\|^{2} ; \quad \forall\|\boldsymbol{e}\| \geq \mu=\frac{2 \lambda_{\max }\left(\boldsymbol{P}_{L}\right)\|\overline{\boldsymbol{\delta}}\|}{\nu \zeta}
\end{aligned}
$$

where $0<\nu<1$. Since $\bar{\delta}$ is bounded and $\boldsymbol{P}_{L}$ exists, we conclude that the solutions of the perturbed system are bounded. Hence, condition 4 of the I\&I stabilization theorem is fulfilled while considering the restrictions on $\nu$ and $\zeta$. With the equations (22), (25), condition 2), and with the equations (27),(29) condition 3 ) of the theorem are fulfilled. Since condition 1) is valid by definition, $\boldsymbol{x}^{*}=\boldsymbol{\pi}\left(\boldsymbol{\xi}^{*}\right)$ is a globally asymptotically stable equilibrium of the closed-loop system (15) with control law (31) that uses the modified rigid body control law (17),(20).

\section{Simulations}

For the simulations an antagonistic joint was considered. The plant parameters and the controller parameters are given in Table II with the friction model $\boldsymbol{f}_{f, \theta}=f_{f, 0} \boldsymbol{x}_{4}$. The sample time was set to $0.1 \mathrm{~ms}$. The I\&I controller is compared with a simple motor PD controller with setpoints that are derived from the desired link position and desired pretension. Both controllers are tested to change the pretension $w_{n}(t)$ from 0 to $5 \sqrt{2} \mathrm{Nm}$ starting at time $t=0 \mathrm{~s}$. At time $t=2 \mathrm{~s}$ a link side motion $\xi_{1}^{*}(t)$ to move sufficiently smooth from $0 \mathrm{rad}$ to $0.2 \mathrm{rad}$ is commanded. The reaction to a first order filtered step (time constant $10 \mathrm{~ms}$ ) in external torque of $\tau_{\text {ext }}=1 \mathrm{Nm}$ on the link was examined at time $t=4 \mathrm{~s}$. The trajectories of these input values are illustrated in Fig. 4. At the end of this section the sensitivity to parameter uncertainty is examined.

\section{A. I\&I Control}

The positions of the link and the motors for this simulation for the I\&I controller are depicted in Fig. 5. In the following presentation then only the tracking errors ${ }^{3}$ will be discussed. At time $t=0 s$, the pretension $w_{n}(t)$ is changed from 0 to $5 \sqrt{2} \mathrm{Nm}$. It can be seen in the figures 5 and 6 that both the motors move in the same direction such that they wind up against each other, and in this way the pretension is realized. Since the tendon stiffness is nonlinear this pretension results

\footnotetext{
${ }^{2}$ This condition can only be fulfilled for sufficiently small values of $\delta_{i}<$ $\left|\delta_{\max }\right|$. This threshold grows by choosing smaller values for the controller parameters $K_{p}, K_{d}$.

${ }^{3}$ The displacements in the motor positions are represented w.r.t. the corresponding desired link position. Therefore, the motor displacements were computed as $\boldsymbol{P}^{T} \xi_{1}^{*}-\boldsymbol{x}_{3}$.
} 


\begin{tabular}{|c|c|c|c|}
\hline \hline$M$ & \multicolumn{2}{|c|}{1} & $\mathrm{kgm}^{2}$ \\
\hline $\boldsymbol{M}_{\theta}$ & $\begin{array}{cc}0.5 & 0 \\
0 & 0.5\end{array}$ & $\mathrm{kgm}^{2}$ \\
\hline $\boldsymbol{P}$ & \multicolumn{2}{|c|}{$-1]$} & $\mathrm{m}$ \\
\hline$\gamma_{i}$ & \multicolumn{2}{|c|}{1} & $1 / \mathrm{rad}$ \\
\hline$k_{t, i}$ & \multicolumn{2}{|c|}{500} & $\mathrm{Nm} / \mathrm{rad}$ \\
\hline$f_{f, 0}$ & 0.001 & $\mathrm{Nms} / \mathrm{rad}$ \\
\hline \hline$K_{p}$ & \multicolumn{2}{|c|}{500} & $\mathrm{Nm} / \mathrm{rad}$ \\
\hline$K_{d}$ & 50 & $\mathrm{Nms} / \mathrm{rad}$ \\
\hline$K_{z, 1}$ & $\begin{array}{cc}100 & 25 \\
25 & 100\end{array}$ & $\mathrm{Nm} / \mathrm{rad}$ \\
\hline$K_{z, 2}$ & $\begin{array}{cc}50 & 5 \\
5 & 50\end{array}$ & $\mathrm{Nms} / \mathrm{rad}$ \\
\hline \hline
\end{tabular}

TABLE II

PLANT AND CONTROLLER PARAMETERS.

as well in an increased mechanical joint stiffness. In the case of the motion command at $t=2 \mathrm{~s}$, the tracking error, that can be observed in Fig. 6, remains below $3 \cdot 10^{-5} \mathrm{rad}$ which demonstrates the excellent tracking capabilities. This error stems from the gains of $\boldsymbol{K}_{z}$. With increasing $\boldsymbol{K}_{z}$ the tracking error decreases. Note that the acceleration feedforward term $M \dot{\xi}_{2}^{*}(t)$ requires sufficiently smooth trajectories. Otherwise, it generates very large peaks for the control input, since its second derivative is used in equation (30). These peaks are generated to minimize the effect of the motor inertia and enables therefore the accurate tracking of the link position.

A filtered step in external torque of $1 \mathrm{Nm}$ was exerted on the link at time $t=4 \mathrm{~s}$. A compliant behavior of the link is observed and behaves accordingly to the target dynamics system (16). For the steady-state the deflection is $0.002 \mathrm{rad}$ that corresponds to the expected $\tau_{e x t} / K_{p}=1 / 500 \mathrm{rad}$. In Fig. 7 the control signal shows the additional component to realize the pretension. The off-the-manifold dynamics converge to zero (c.f. Fig. 8) and therefore the required virtual motor positions are realized. Note that for this parametrization the term $\delta$ stay by far below the threshold $\left|\delta_{\max }\right| \approx 0.164$.

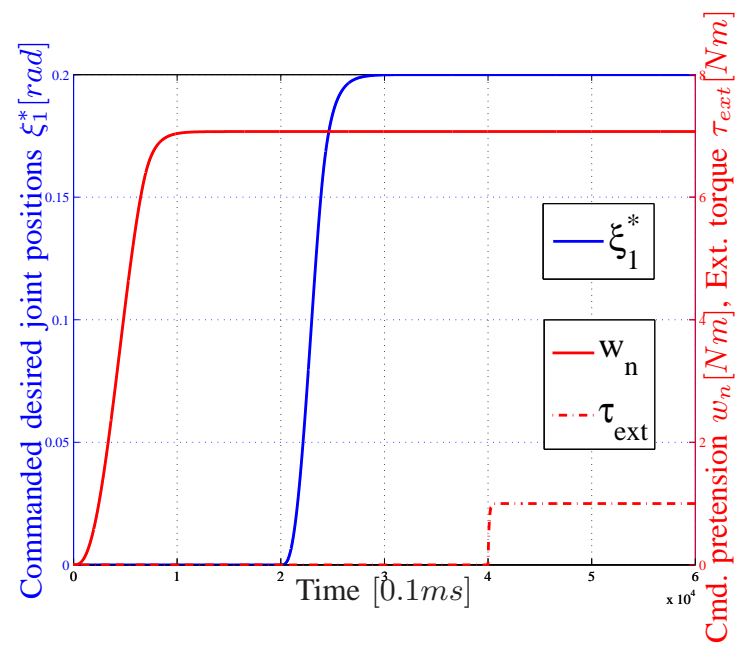

Fig. 4. Trajectories of the commanded desired joint positions, of the pretension, and of the external torque.

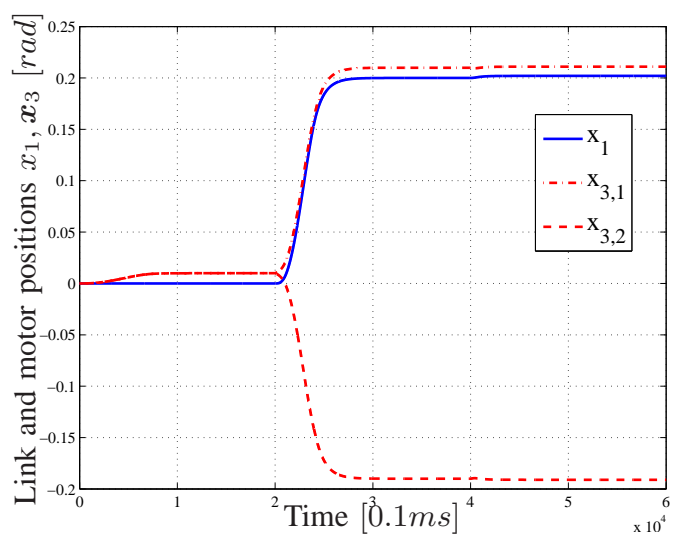

Fig. 5. I\&I control: Link and motor positions. Note that in the pretension phase both motors move in the same direction while moving in the opposite direction in the case of moving the link position. The external torque displaces the link position as specified in the target dynamics. At the same time the motor positions are displaced as well.

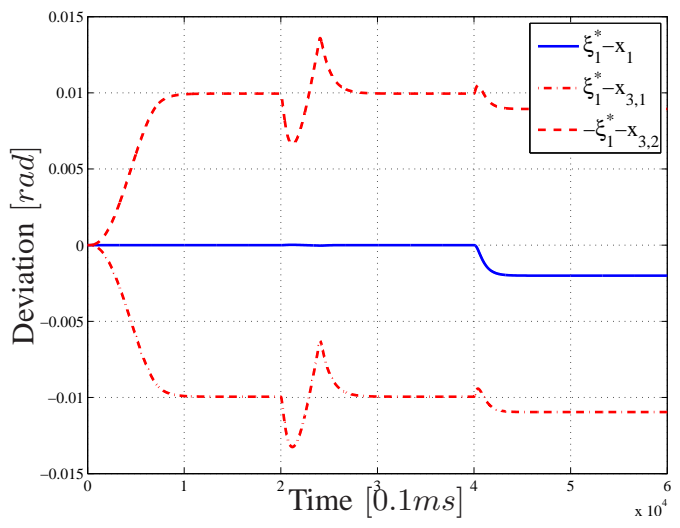

Fig. 6. I\&I control: Position error for link and motor positions.

\section{B. Comparison with simple PD motor control}

The performance of the controller is compared with a simple motor PD controller with setpoints that are derived from the desired link position and desired pretension

$$
\begin{gathered}
\boldsymbol{f}_{m}=-\boldsymbol{K}_{p, m} \boldsymbol{e}_{m}-\boldsymbol{K}_{d, m} \dot{\boldsymbol{e}}_{m}+\boldsymbol{M}_{m} \ddot{\boldsymbol{x}}_{3, d}+\boldsymbol{f}_{f, \theta}+\mathcal{N}(\boldsymbol{P}) \boldsymbol{w}_{n} \text { (52) } \\
\text { with } \boldsymbol{e}_{m}=\boldsymbol{x}_{3}-\boldsymbol{P}^{T} \boldsymbol{x}_{3, d}, \quad \text { and } \boldsymbol{K}_{p, m}=
\end{gathered}
$$
$\operatorname{diag}\{250,250\} \mathrm{Nm} / \mathrm{rad}, \boldsymbol{K}_{d, m}=\operatorname{diag}\{25,25\} \mathrm{Nms} / \mathrm{rad}$ the motor stiffness and the motor damping matrices ${ }^{4}$, respectively. The inertia matrix for the feedforward of the acceleration term $\boldsymbol{M}_{m}$ is defined by the sum of the motor inertia $\boldsymbol{M}_{\theta}$ and the reflected joint inertia $\boldsymbol{P}^{+} M \boldsymbol{P}^{+T}$. The desired motor positions are computed by $\boldsymbol{x}_{3, i, d}=\boldsymbol{P}^{T} \xi_{1}^{*}+\gamma_{i}^{-1} \ln \left(1-k_{t, i}^{-1} w_{n}\right)$. In Fig. 9 it can be seen that the pretension is well adjusted. It can be further observed that the tracking error is by several magnitudes larger than the one of the I\&I controller. The corresponding control signal $f_{m}$ is depicted in Fig. 10. Parallel to this controller we computed the off-the-manifold dynamics that are shown in Fig. 11 in which weakly damped oscillations

\footnotetext{
${ }^{4}$ The motor controller gains have been chosen to fulfill the equations $K_{p}=\boldsymbol{P} \boldsymbol{K}_{p, m} \boldsymbol{P}^{T}$ and $K_{d}=\boldsymbol{P} \boldsymbol{K}_{d, m} \boldsymbol{P}^{T}$.
} 


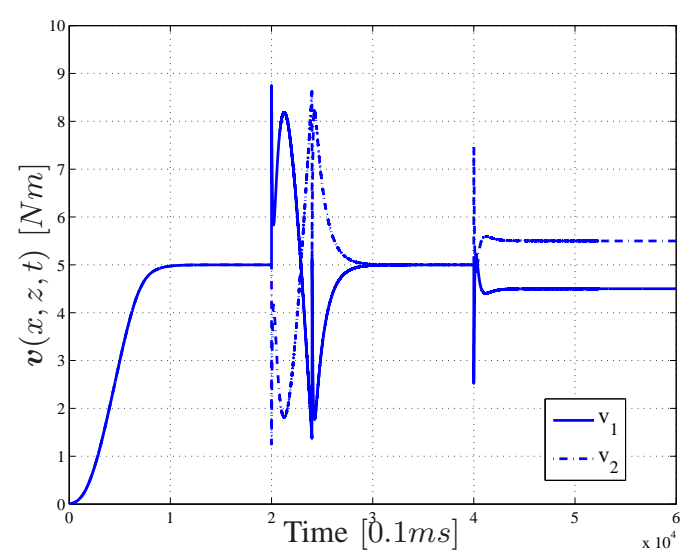

Fig. 7. I\&I control: Control input $\boldsymbol{v}(x, z, t)$. Note there is a peak at $t=4.0 \mathrm{~s}$ which is due to $\dot{\tau}_{\text {ext }}$ that appears in the controller.

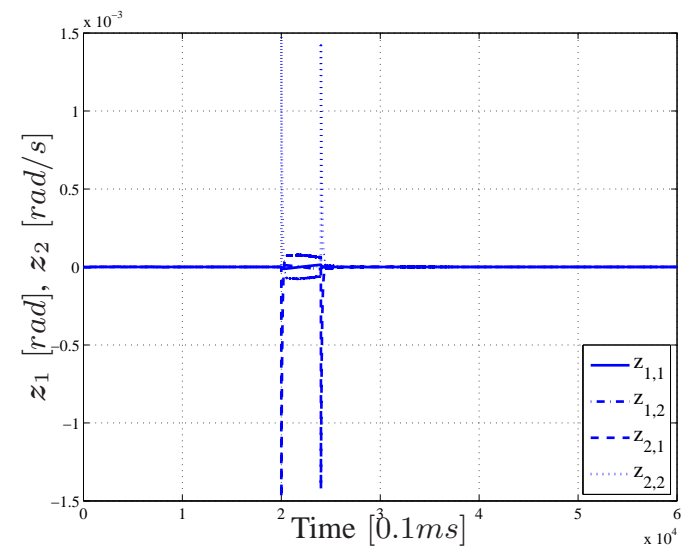

Fig. 8. I\&I control: Off-the-manifold dynamics $\boldsymbol{z}$.

can be observed which are quickly damped in the case of the I\&I controller. These vibrations can also be seen in figure 9 in the time interval $t=[2.5 \mathrm{~s} \ldots 3.0 \mathrm{~s}]$.

\section{Sensitivity Analysis}

The same task was performed again for the I\&I controller, whereas the friction parameter, that is often not well known in practice, was replaced by an estimate $\hat{f}_{f, 0}=1.3 f_{f, 0}$, and at the same time the motor inertia was assumed to be $\hat{M}_{\theta}=$ $0.7 M_{\theta}$ in order to study the sensitivity of the controller to parameter errors. Note, that the friction compensation term $\boldsymbol{f}_{f, \theta}$ appears in the control law (31). In Fig. 12 it can be seen that the tracking error and the settling time is increased in the case of the link motion command at $t=2.0 \mathrm{~s}$. This indicates a robust behavior w.r.t. parameter uncertainties. In comparison the simulation results are shown for the case of the simple motor controller in Fig. 13. From that we defer that even in the case of (reasonable) parameter uncertainties the I\&I controller has a better tracking performance.

\section{CONCLUSION}

In this paper the Immersion and Invariance controller was applied to an antagonistic joint with nonlinear mechanical

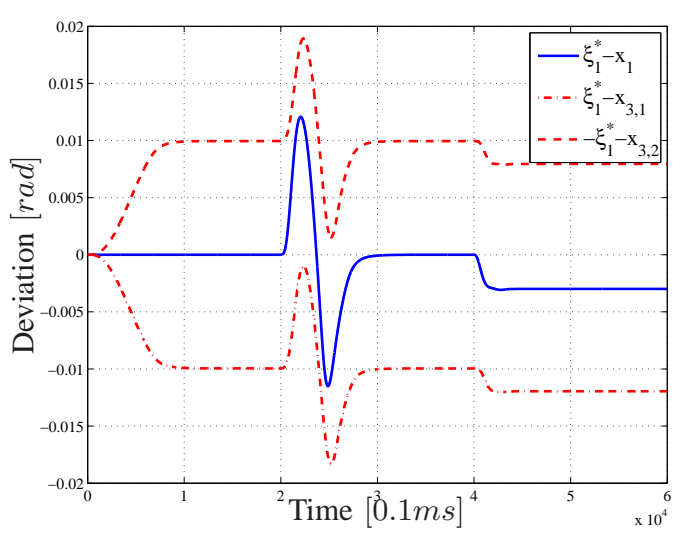

Fig. 9. Motor PD control: Position error for link and motor position.

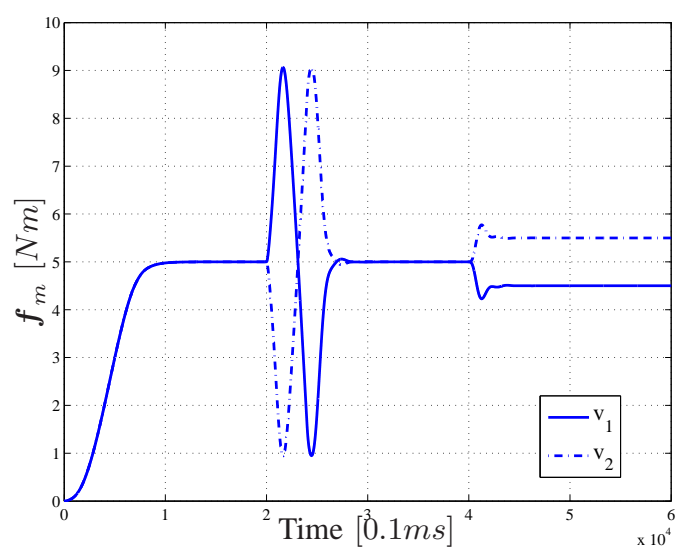

Fig. 10. Motor PD control: Control input $\boldsymbol{v}=\boldsymbol{f}_{m}$.

stiffness. The model of a tendon controlled variable stiffness mechanism was briefly introduced. The target system was specified to behave as desired second order impedance while at the same time a pretension was commanded to the tendon forces that lie in the nullspace of the tendon coupling matrix. In this way, we were able to assign on the one side the controlled impedance behavior for the link and at the same time set the mechanical stiffness of the mechanism by varying the pretension. Furthermore, we discussed the physical meanings of the map $\pi$ that corresponds to virtual desired motor positions and that the off-the-manifold stabilization represents an underlying motor position controller. The I\&I controller was compared in simulations with a simple motor PD based controller. The I\&I controller clearly outperforms the motor PD controller and the off-the-manifold coordinates are well damped and converge to zero - even in the case of parameter uncertainties. As future work we plan to implement this controller on hardware and to expand to the case of multiple degrees of freedom. Furthermore, we consider to use a reduced-order observer for the robotic system.

\section{ACKNOWLEDGEMENT}

This work has been partially funded by the European Commissions Seventh Framework Programme as part of 


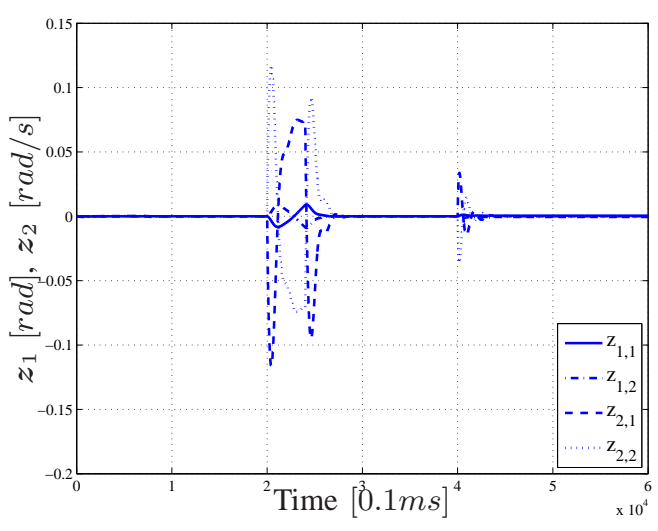

Fig. 11. Motor PD control: Off-the-manifold dynamics $\boldsymbol{z}$.

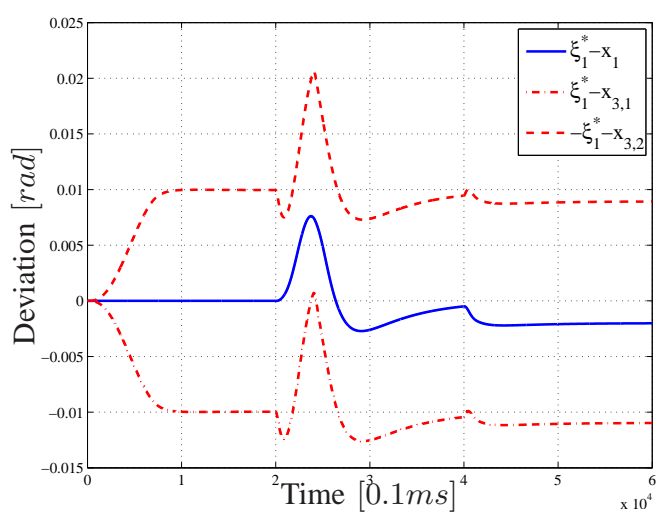

Fig. 12. I\&I control: Position error for link and motor position with $\hat{f}_{f, 0}=$ $1.3 f_{f, 0}$ and $\hat{M}_{\theta}=0.7 M_{\theta}$.

the projects VIACTORS (grant no. 231554) and The Hand Embodied (grant no. 248587).

\section{REFERENCES}

[1] K. Laurin-Kovitz, J. E. Colgate, and S. D. R. Carnes, "Design of programmable passive impedance," in IEEE International Conference on Robotics and Automation, 1991, pp. 1476-1481.

[2] C. English and D. Russell, "Implementation of variable joint stiffness through antagonistic actuation using rolamite springs," Mechanism and Machine Theory, vol. 34, no. 1, pp. 27-40, 1999.

[3] S. A. Migliore, E. A. Brown, and S. P. DeWeerth, "Biologically inspired joint stiffness control," in IEEE International Conference on Robotics and Automation, 2005.

[4] B. Vanderborght, B. Verrelst, R. V. Ham, M. V. Damme, D. Lefeber, B. M. Y. Duran, and P. Beyl, "Exploiting natural dynamics to reduce energy consumption by controlling the compliance of soft actuators," The International Journal of Robotics Research, vol. 25, no. 4, pp. 343-358, 2006.

[5] G. Palli, C. Melchiorri, T. Wimböck, M. Grebenstein, and G. Hirzinger, "Feedback linearization and simultaneous stiffness-position control of robots with antagonistic actuated joints," in IEEE International Conference on Robotics and Automation, 2007, pp. 4367-4372.

[6] S. Wolf and G. Hirzinger, "A new variable stiffness design: Matching requirements of the next robot generation," in IEEE International Conference on Robotics and Automation, 2008.

[7] M. Grebenstein and P. van der Smagt, "Antagonism for highly anthropomorphic hand-arm system," Advanced Robotics, vol. 22, pp. 39-55, 2008.

[8] W. Townsend, "The effect of transmission design on force-controlled manipulator performance," Ph.D. dissertation, Massachusetts Institute of Technology, Cambridge, MA, USA, 1988

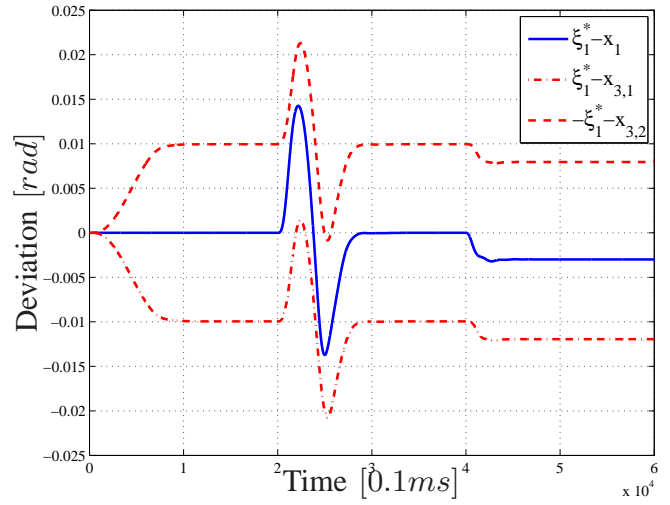

Fig. 13. Motor PD control: Position error for link and motor position with $\hat{f}_{f, 0}=1.3 f_{f, 0}$ and $\hat{M}_{\theta}=0.7 M_{\theta}$.

[9] W. Townsend and J. Salisbury, "Mechanical bandwidth as a guideline to high-performance manipulator design," in IEEE International Conference on Robotics and Automation, 1989.

[10] S. Jacobsen, J. Wood, D. Knutti, and K. Biggers, "Utah/mit dextroushand - work in progress," International Journal of Robotics Research, vol. 3, no. 4, pp. 21-50, 1984.

[11] S. Jacobsen, H. Ko, E. Iversen, and C. Davis, "Antagonistic control of a tendon driven manipulator," in IEEE International Conference on Robotics and Automation, 1989.

[12] J. Salisbury and M. T. Mason, "Robot hands and the mechanics of manipulation," MIT Press, 1985.

[13] S. Marcheschi, A. Frisoli, C. A. Avizzano, and M. Bergamasco, "A method for modeling and control complex tendon transmissions in haptic interfaces," in IEEE International Conference on Robotics and Automation, 2005.

[14] S. B. M. Okada and Y. Nakamura, "Skill of compliance with controlled charging/discharging of kinetic energy," in IEEE International Conference on Robotics and Automation, 2002.

[15] G. Tonietti, R. Schiavi, and A. Bicchi, "Design and control of a variable stiffness actuator for safe and fast physical human/robot interaction," in IEEE International Conference on Robotics and Automation, 2005, pp. 528-533.

[16] H. Kobayashi and R. Ozawa, "Adaptive neural network control of tendon-driven mechanisms with elastic tendons," Automatica, vol. 39, pp. 1509-1519, 2003.

[17] G. Palli, "Model and control of tendon actuated robots," Ph.D. dissertation, DEIS, University of Bologna, 2007.

[18] Ch. Ott, A. Albu-Schäffer, A. Kugi, and G. Hirzinger, "On the passivity based impedance control of flexible joint robots," IEEE Transactions on Robotics, vol. 24, no. 2, pp. 416-429, April 2008.

[19] A. Albu-Schäffer, Ch. Ott, and G. Hirzinger, "A unified passivity based control framework for position, torque and impedance control of flexible joint robots," International Journal of Robotics Research, vol. 26, no. 1, pp. 23 - 39, January 2007.

[20] T. Wimböck, Ch. Ott, A. Albu-Schäffer, A. Kugi, and G. Hirzinger, "Impedance control for variable stiffness mechanisms with nonlinear joint coupling," in IEEE/RSJ International Conference on Intelligent Robots and Systems, 2008, pp. 3796-3803.

[21] A. Astolfi, D. Karagiannis, and R. Ortega, Nonlinear and Adaptive Control with Applications, 1st ed. Springer-Verlag, 2008.

[22] R. Murray, Z. Li, and S. Sastry, A Mathematical Introduction to Robotic Manipulation. CRC Press, 1994.

[23] H. Kobayashi, K. Hyodo, and D. Ogane, "On tendon-driven robotic mechanisms with redundant tendons," The International Journal of Robotics Research, vol. 17, no. 4, pp. 561-571, 1998.

[24] J. Slotine and W. Li, "On the adaptive control of robot manipulators," International Journal of Robotics Research, vol. 6, no. 3, pp. 49-59, 1987.

[25] H. K. Khalil, Nonlinear Systems, 3rd ed. Prentice Hall, 2002. 